\title{
OCTAHEDRAL STRUCTURE OF THE HIROTA-MIWA EQUATION
}

\author{
SATORU SAITO \\ 4-19-10 Hakusan, Midoriku \\ Yokohama 266-0006, Japan \\ saito_ru@nifty.com \\ Received 18 July 2012 \\ Accepted 28 August 2012 \\ Published 31 December 2012
}

\begin{abstract}
The Hirota-Miwa equation is studied from the view point of derived category.
Keywords: Hirota-Miwa equation; derived category; singularity confinement.

Mathematics Subject Classification 2000: 22E46, 53C35, 57S20
\end{abstract}

\section{Introduction}

There have been known many models in physics, including the theory of strings, which are described by means of the theory of KP hierarchy. The Hirota-Miwa (HM) equation $[6,11]$ itself, from which all equations of the KP hierarchy can be derived, has been studied in various respects of mathematics, such as algebraic geometry, representation theory of algebra, conformal field theory, and so on.

On the other hand, from the pure mathematical view point, the theory of sheaf category has been developed extensively to unify all fields in mathematics [3, 4] and [10]. ${ }^{\text {a }}$ In particular, since it was combined with the theory of strings more than two decades ago $[2,9]$, the theory of derived category has been investigated intensively.

The purpose of this note is to point out that the theory of KP hierarchy can be described in terms of the theory of derived category. To this end we study in detail the HM equation $[6,11]$, such that various features of a triangle category become manifest, even though mathematical details will not be given. If the KP hierarchy is embedded successfully in the theory of derived category we should be able to characterize integrable systems both in physics and mathematics in a common base.

In order to introduce the notion of category to the theory of KP hierarchy, we first study, in $\S 2$, the Bäcklund transformation of the HM equation [15, 21]. It will be accomplished by the use of exterial "difference" form $d_{B}$ in the lattice space $\mathbb{Z}^{4}$. This operator connects

\footnotetext{
${ }^{\mathrm{a}}$ There exist many text books on the subject, but the author learned much from this thesis.
} 
one octahedron to another, on which six $\tau$ functions of the HM equation are attached at every corner. An octahedron is on a hyperplane in $\mathbb{Z}^{4}$. Hence $d_{B}$ shifts an octahedron in one hyperplane to another. We can consider an octahedron and the Bäcklund transformation as a pair of an object and a morphism of category. The exactness of $d_{B}$, which owes to the Grassmann nature of vertex operators [14], naturally provides us the structure of a complex of this category.

In $\S 3$ we consider the local feature of the octahedron. If we associate a morphism to every edge of the octahedron we can study the flow of information from one vertex to another. In other words the objects in this case are the $\tau$ functions at corners of the octahedron. We show in the text that the flow of information in the procedure of solving the HM equation exactly fits the axioms of the triangle category. This also means that every step of solving the HM equation starting from an initial value forms a complex. Notice that this complex is in one hyperplane of $\mathbb{Z}^{4}$. If we combine this complex together with the complex of the Bäcklund transformation, we obtain a double complex, as illustrated in Fig. 3.

The localization properties of the complexes have to be considered if we argue the derived categories. We show in this note that it is realized by the generation of the invariant varieties of periodic points (IVPP) [16-18, 20] after liberated from the singularity confinement [19]. In fact we can follow explicitly, in the case of Lotka-Volterra map, how the localization of the category takes place and generates the IVPPs. Section 5 is devoted to conclusion.

\section{Global Feature of the HM Equation}

The purpose of this section is to reformulate the theory of KP hierarchy in such a way that the structure of complex becomes manifest.

\subsection{Hirota-Miwa equation}

It is well known that all integrable maps which belong to the $\mathrm{KP}$ hierarchy can be derived from the following single HM equation $[6,11]$, defined by

$$
\begin{aligned}
& a_{14} a_{23} f\left(p_{1}+1, p_{2}, p_{3}, p_{4}+1\right) f\left(p_{1}, p_{2}+1, p_{3}+1, p_{4}\right) \\
& \quad-a_{13} a_{24} f\left(p_{1}, p_{2}+1, p_{3}, p_{4}+1\right) f\left(p_{1}+1, p_{2}, p_{3}+1, p_{4}\right) \\
& \quad+a_{12} a_{34} f\left(p_{1}, p_{2}, p_{3}+1, p_{4}+1\right) f\left(p_{1}+1, p_{2}+1, p_{3}, p_{4}\right)=0 .
\end{aligned}
$$

Here $f(\boldsymbol{p}) \in \mathbb{C}$ is a function of $\boldsymbol{p}=\left(p_{1}, p_{2}, p_{3}, p_{4}\right) \in \mathbb{Z}^{4}$ and $a_{i j}=-a_{j i} \in \mathbb{C}$ are arbitrary constants.

The integrability of (2.1) enables us to determine $f(\boldsymbol{p})$ for all $\boldsymbol{p} \in \mathbb{Z}^{4}$. In fact the general solution of (2.1), which is called the $\tau$ function, is given by

$$
\tau\left(p_{1}, p_{2}, \ldots, p_{N}, z_{1}, z_{2}, \ldots, z_{N}\right):=\frac{\left\langle 0\left|V\left(p_{1}, z_{1}\right) V\left(p_{2}, z_{2}\right) \cdots V\left(p_{N}, z_{N}\right)\right| D\right\rangle}{\left\langle 0\left|V\left(p_{1}, z_{1}\right) V\left(p_{2}, z_{2}\right) \cdots V\left(p_{N}, z_{N}\right)\right| 0\right\rangle},
$$

where $V(p, z)$ is the vertex operator with momentum $p$ attached at $z$ on a Riemann surface [14]. The state $|D\rangle$ specifies the Riemann surface, while $|0\rangle$ denotes the empty state. The $\tau$ function is nothing but the Plücker coordinate, which determines four-dimensional subspace in the Grassmannian parametrized by $\left(z_{1}, z_{2}, \ldots, z_{N}\right)$. 
An important property of this function owes to the Grassmann nature of the vertex operators when the magnitude of the momentum is unity. Namely, when $p= \pm 1$ the vertex operators $\psi(z):=V(1, z)$ and $\bar{\psi}(z):=V(-1, z)$ satisfy [14]

$$
\psi(z) \psi(z)=0, \quad \bar{\psi}(z) \bar{\psi}(z)=0 .
$$

\subsection{Exterior difference form}

We reformulate the Bäcklund transformation of the HM equation, which was discussed in $[15,21]$, in this and the next subsections, such that the structure of complex of the transformation becomes manifest.

In order to study the nature of solutions of the HM equation (2.1), it is convenient to introduce the notations

$$
D_{j} f(\boldsymbol{p}):=f\left(\boldsymbol{p}+\boldsymbol{\delta}_{j}\right), \quad\left(\boldsymbol{\delta}_{j}\right)_{i}:=\delta_{i j}
$$

and

$$
\begin{gathered}
f_{i}(\boldsymbol{p}):=D_{i} f(\boldsymbol{p}), \quad f_{i j}(\boldsymbol{p}):=D_{i} D_{j} f(\boldsymbol{p}) \\
f_{(i)}(\boldsymbol{p}):=D_{k} D_{l} D_{m} f(\boldsymbol{p}), \quad f_{(i j)}(\boldsymbol{p}):=D_{k} D_{l} f(\boldsymbol{p}), \quad k, l, m \neq i, j
\end{gathered}
$$

to simplify our arguments. We define an "exterior difference operator" $d_{B}$ to obtain the "difference one form" $\boldsymbol{f}$ from a function $f(\boldsymbol{p})$ by

$$
\boldsymbol{f}:=d_{B} f(\boldsymbol{p}):=\sum_{j=1}^{4} D_{j} f(\boldsymbol{p}) d p_{j}=\sum_{j=1}^{4} f_{j}(\boldsymbol{p}) d p_{j} .
$$

The formula

$$
d_{B} \boldsymbol{f}=d_{B}^{2} f(\boldsymbol{p})=\sum_{i, j=1}^{4} f_{i j} d p_{i} \wedge d p_{j} \equiv 0
$$

follows immediately, such that the condition (2.3) is naturally satisfied.

If we define the "difference two forms" by

$$
F:=\sum_{i, j}^{4} a_{i j} f_{i j} d p_{i} \wedge d p_{j}, \quad \tilde{F}:=\sum_{i, j}^{4} a_{(i j)} f_{i j} d p_{i} \wedge d p_{j},
$$

each of the next formulae is equivalent to the HM equation (2.1):

$$
\operatorname{det}\left\{F_{i j}\right\}=0, \quad \operatorname{det}\left\{\tilde{F}_{(i j)}\right\}=0 .
$$

We notice that the Eq. (2.1), or (2.5), determines a relation among six $f_{i j}$ 's which are on a three-dimensional hyperplane defined by $p_{1}+p_{2}+p_{3}+p_{4}+2=t$ (= constant). If we denote the hyperplane by $\Xi_{t}$, and introduce the three-dimensional coordinates $\left(k_{1}, k_{2}, k_{3}\right)$ by

$$
k_{1}:=\frac{p_{1}-p_{2}-p_{3}+p_{4}}{2}, \quad k_{2}:=\frac{p_{2}-p_{3}-p_{1}+p_{4}}{2}, \quad k_{3}:=\frac{p_{3}-p_{1}-p_{2}+p_{4}}{2}
$$

they are on the vertexes of an octahedron on $\Xi_{t}$ as illustrated in Fig. 1.

Octahedra fill, together with tetrahedra, this three-dimensional lattice space. Every octahedron is connected with other 12 octahedra by edges. All points of this three-dimensional 


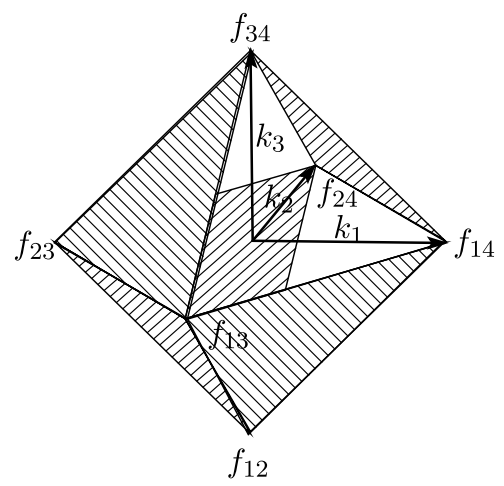

Fig. 1.

lattice space, however, are not the points of the same hyperplane $\Xi_{t}$, specified by $t$. The centers of the octahedra are on $\Xi_{t-2}$, while the centers of tetrahedra are on $\Xi_{t \pm 1}$. We notice that $f_{j}$ 's and $f_{(j)}$ 's are on $\Xi_{t \pm 1}$. Hence the operator $d_{B}$ moves points on $\Xi_{t}$ to those of $\Xi_{t+1}$.

It will be worthwhile to mention that there are many other integrable discrete equations of octahedron type. Since they have been well classified in detail [1] we should be able to study them in a way we discuss below.

\subsection{Bäcklund transformation}

Let $f(\boldsymbol{p})$ be a solution of $(2.5)$ and $\boldsymbol{g}=d_{B} g(\boldsymbol{p})$ be a difference one form which satisfies the linear equation

$$
\tilde{F} \wedge \boldsymbol{g}=0
$$

We can rewrite the three form $\tilde{F} \wedge \boldsymbol{g}$, by using ${ }^{*} d p_{i}:=\epsilon_{i j k l} d p_{j} \wedge d p_{k} \wedge d p_{l}$, as

$$
(\tilde{F} \wedge \boldsymbol{g})_{i}=\sum_{j, k, l=1}^{4} a_{(k l)} f_{k l} g_{j} d p_{k} \wedge d p_{l} \wedge d p_{j}=\sum_{j=1}^{4} a_{i j} f_{(i j)} g_{j}{ }^{*} d p_{i}, \quad i=1,2,3,4 .
$$

The last expression of this formula admits to write (2.6) as

$$
\sum_{j=1}^{4} \tilde{F}_{(i j)} g_{j}=0, \quad i=1,2,3,4 .
$$

It also shows that $\tilde{F} \wedge \boldsymbol{g}$ is not exact. Therefore, for (2.6) to hold, we must require $d_{B}(\tilde{F} \wedge \boldsymbol{g})$ to vanish. Since we can write

$$
d_{B}(\tilde{F} \wedge \boldsymbol{g})=d_{B} \sum_{j=1}^{4} a_{i j} f_{(i j)} g_{j}{ }^{*} d p_{i}=\sum_{j} a_{i j} f_{(j)} g_{i j} d p_{i} \wedge^{*} d p_{i}=\sum_{j} G_{i j} f_{(j)},
$$

we see that (2.6), hence (2.8), is compatible only if

$$
\sum_{j=1}^{4} G_{i j} f_{(j)}=0, \quad i=1,2,3,4
$$

is satisfied. 
We are now ready to describe the Bäcklund transformation of the HM equation in terms of the difference geometry. First we notice that, owing to (2.5), both linear equations (2.8) and (2.9) have nontrivial solutions iff $f(\boldsymbol{p})$ and $g(\boldsymbol{p})$ are solutions of the HM equation. We call (2.8) and (2.9) the linear Bäcklund transformation [15] because they are a set of linear equations which enables us to derive a series of solutions of the HM equation as follows.

(1) Let $f^{(0)}(\boldsymbol{p})$ be a solution of $\operatorname{det}\left(\tilde{F}_{(i j)}\right)=0$ on $\Xi_{t}$.

(2) Substitute $f^{(0)}(\boldsymbol{p})$ into (2.8) and solve it for $\left\{g_{j}\right\}$.

(3) Define $G_{i j}:=a_{i j} D_{i} g_{j}$, then $\operatorname{det}\left(G_{i j}\right)=0$ is satisfied.

(4) Let $g^{(1)}(\boldsymbol{p})$ be the function determined by $G_{i j}$ on $\Xi_{t+1}$.

(5) Substitute $g^{(1)}(\boldsymbol{p})$ into (2.9) and solve it for $\left\{f_{(j)}\right\}$.

(6) Define $\tilde{F}_{i j}:=a_{(i j)} D_{i} f_{(j)}$, then $\operatorname{det}\left(\tilde{F}_{i j}\right)=0$ is satisfied.

(7) Let $f^{(2)}(\boldsymbol{p})$ be the function determined by $\tilde{F}_{i j}$ on $\Xi_{t+2}$.

We can repeat this procedure to obtain a sequence

$$
f^{(0)} \stackrel{d_{B}}{\longrightarrow} g^{(1)} \stackrel{d_{B}}{\longrightarrow} f^{(2)} \stackrel{d_{B}}{\longrightarrow} g^{(3)} \stackrel{d_{B}}{\longrightarrow} \cdots
$$

of solutions of the HM equation. It is important to mention that, since (2.9) is obtained from (2.8) by an operation of $d_{B}$, the hyperplanes in the four-dimensional lattice space, on which octahedra are associated, are different. This also means that at every step of the transformation we obtain a new solution of the HM equation. Since a solution determines an octahedron the above sequence of the Bäcklund transformation also implies a scheme of transformations of octahedra

$$
\mathcal{O}^{(0)} \stackrel{d_{B}}{\longrightarrow} \mathcal{O}^{(1)} \stackrel{d_{B}}{\longrightarrow} \mathcal{O}^{(2)} \stackrel{d_{B}}{\longrightarrow} \mathcal{O}^{(3)} \stackrel{d_{B}}{\longrightarrow} \cdots .
$$

The structure of complex is manifest in this diagram such that $\left(\mathcal{O}^{(j)}, d_{B}\right)$ forms a pair of an object and morphism of the category. We say this is a global feature of the HM equation in the sense that all information in one three-dimensional hyperplane is transformed by $d_{B}$ to another hyperplane at once.

\section{Local Feature of the HM Equation}

We would like to know, in this section, how a local information propagates to other parts on one three-dimensional hyperplane under the control of the HM equation.

\subsection{Translation of octahedra}

In order to explore the mechanism how the information of an octahedron transfers to other octahedra, we define the translation operator by

$$
T:=\prod_{j=1}^{4} D_{j}^{m_{j}}: f(\boldsymbol{p}) \rightarrow f(\boldsymbol{p}+\boldsymbol{m}), \quad m_{j}=0, \pm 1 .
$$

The values of $m_{j}$ are strictly constrained by the condition (2.3). Needless to say, $T$ itself satisfies $T^{2}=0$.

Information of an octahedron will be transferred properly only when the octahedron is translated by $T$ to a top of another octahedron. If we denote $\sum_{j=1}^{4} m_{j}$ by $m$, it can change 
from -4 to 4 . When $m= \pm 4$, the octahedron is moved to itself in the three-dimensional lattice space, since the values of $\left(k_{1}, k_{2}, k_{3}\right)$ remain the same. In the four-dimensional lattice space, however, it is moved to the $\Xi_{t \pm 4}$ hyperplane. Similarly, when $m \neq 0$, all points are moved to the hyperplane $\Xi_{t+m}$. From our arguments in the previous section the octahedra translated to other hyperplanes must belong to other solutions.

When $m=0$, the translation is constrained on $\Xi_{t}$. In other words the information of a solution can propagate to other places of the same $\Xi_{t}$. There are 12 possible operators which satisfy $m=0$,

$$
T_{j i}:=D_{j} D_{i}^{-1}, \quad j \neq i
$$

among which only six are independent because $T_{j i}=T_{i j}^{-1}$. As we apply $T_{i j}$ to an octahedron, the octahedron itself is translated to its neighbor in the direction of $p_{i}-p_{j}$. Every pair of octahedra connected by $T_{i j}$ has an edge in common, as illustrated in Fig. 2.

\subsection{Octahedral axiom}

In addition to the translation of an octahedron the operator $T_{j i}$ plays another important role in the octahedron. Namely every vertex of the octahedron is locally connected with others by this operator. To understand the mechanism how the information is transferred to other octahedra, we must know in detail how the vertexes are connected within the octahedron.

Let us denote by

$$
\tilde{S}_{j}=(X, Y, Z):=\left(f_{b c}, f_{c a}, f_{a b}\right), \quad a, b, c \neq j, j=1,2,3,4
$$

the four triangles of the octahedron as shown in Fig. 2, and by

$$
S_{j}=\left(X^{\prime}, Y^{\prime}, Z^{\prime}\right):=\left(f_{j a}, f_{j b}, f_{j c}\right), \quad a, b, c \neq j, j=1,2,3,4
$$

those parallel to them. The operator $T_{j i}$ connects the corners of the triangle $S_{j}$. We define the order of vertexes by $c \rightarrow b \rightarrow a \rightarrow c$ when $c<b<a$. Then every triangle $S_{j}$ of Fig. 2 is

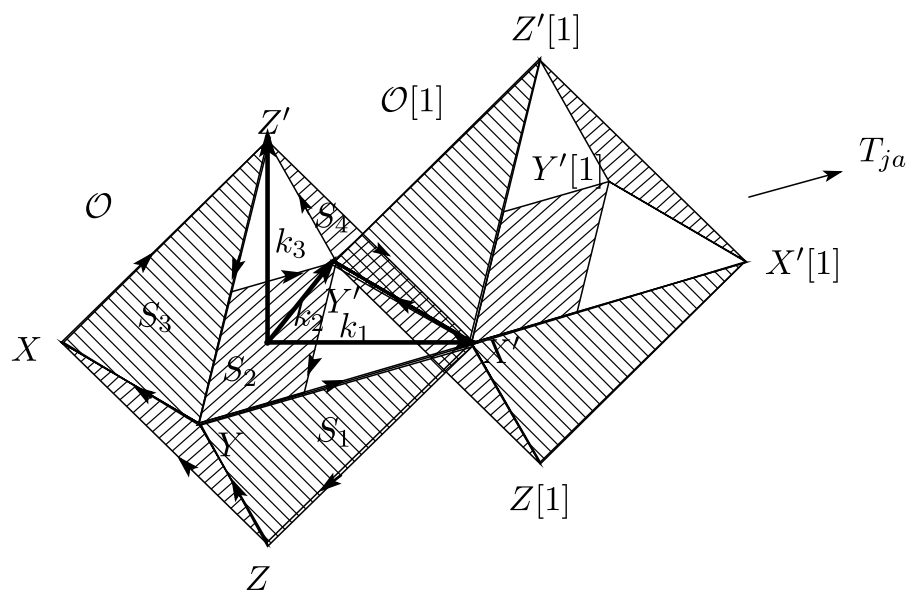

Fig. 2. 
oriented and the chain of the translation along the arrows

$$
f_{j c} \stackrel{T_{b c}}{\longrightarrow} f_{j b} \stackrel{T_{a b}}{\longrightarrow} f_{j a} \stackrel{T_{c a}}{\longrightarrow} f_{j c}
$$

is closed. We should mention that the corresponding triangle $\tilde{S}_{j}$ is unoriented, thus has no face.

As we fix $j$, the octahedron can be specified by two triangles, $\mathcal{O}=\left(\tilde{S}_{j}, S_{j}\right)$. The information of this octahedron $\mathcal{O}$ can be transferred to one neighbor $\mathcal{O}[1]$ by an operator, say $T_{j a}$, as

$$
\left(\tilde{S}_{j}, S_{j}\right) \stackrel{T_{j a}}{\longrightarrow}\left(\tilde{S}_{j}, S_{j}\right)[1] .
$$

We can summarize the flow of information as follows:

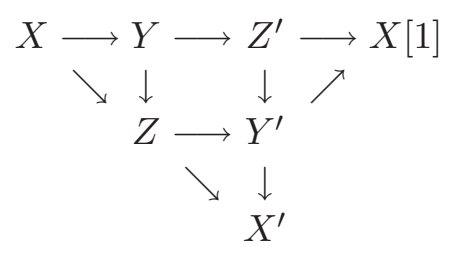

$X[1]$ denotes $X$ in $\mathcal{O}[1]$. It is important to mention that this flow of information is exactly the same with the octahedron axiom in the theory of triangle categories $[2,3,10]$.

\subsection{Double complex}

Because of the fact $T_{j a}^{2}=0$, this procedure cannot be continued, if the contents of $\mathcal{O}$ and $\mathcal{O}[1]$ are the same. In solving initial value problems of a discrete map, however, we must set up new values at every step of the iteration of the map. Let us see, in detail, what happens in the case of the HM equation.

The HM equation (2.1) relates the $f$ 's of $\tilde{S}_{j}$ to those of $S_{j}$ in a bilinear form,

$$
\tilde{S}_{j} \stackrel{H M}{\longrightarrow} S_{j}
$$

Since the triangles $S_{j}$ of $\mathcal{O}$ and $\tilde{S}_{j}[1]$ of $\mathcal{O}[1]$ share one edge, their two vertexes must coincide:

$$
f_{j b}=f_{a b}[1], \quad f_{j c}=f_{a c}[1] .
$$

When we solve the HM equation $\tilde{S}_{j}[1] \stackrel{H M}{\longrightarrow} S_{j}[1]$ in $\mathcal{O}[1]$, we must use these new values in $\tilde{S}_{j}[1]$. Now we can continue this procedure and see how the information transfers along the chain:

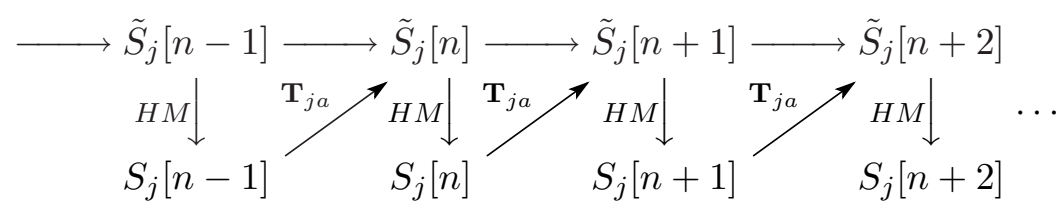

or, simply,

$$
\cdots \stackrel{T_{j a}}{\longrightarrow} \mathcal{O}[n-1] \stackrel{T_{j a}}{\longrightarrow} \mathcal{O}[n] \stackrel{T_{j a}}{\longrightarrow} \mathcal{O}[n+1] \stackrel{T_{j a}}{\longrightarrow} \cdots
$$


If we combine this chain (3.4) together with the sequence of the Bäcklund transformation (2.10) we obtain the following double complex:

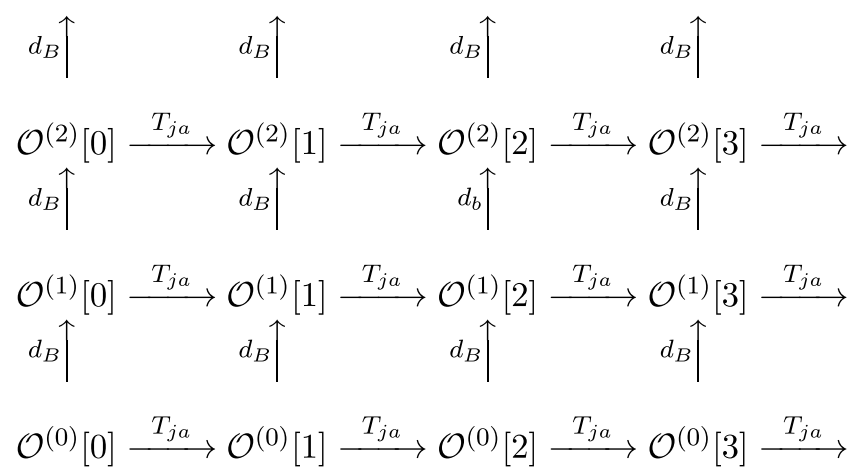

Fig. 3.

\section{Localization and Singularity Confinement}

Our purpose of this section is to study the results of the previous section explicitly and see how information of an octahedron propagates on the chain (3.4) to a remote site.

\subsection{Reduction of the map}

In (3.4) all octahedra are on one hyperplane with $p_{1}+p_{2}+p_{3}+p_{4}$ being fixed. Since the hyperplane is symmetric we can choose $T_{j a}$ by hand, along which information transfers. Let us fix it to $T_{43}$. It is the direction of $k_{1}+k_{2}$ as illustrated in Fig. 2. For simplicity we consider a subspace of the three-dimensional lattice such that only two variables

$$
n:=p_{4}=\frac{2}{3}\left(k_{1}+k_{2}+k_{3}\right), \quad q:=p_{1}-p_{2}=k_{1}-k_{2}
$$

are free. If we define $\tau_{q}^{(n)}:=f_{4}(\mathbf{p})$ and specify the coefficients properly, Eq. (2.1) becomes

$$
\tau_{q-1}^{(n-1)} \tau_{q+1}^{(n)}-2 \tau_{q+1}^{(n-1)} \tau_{q-1}^{(n)}+\tau_{q}^{(n-1)} \tau_{q}^{(n)}=0 .
$$

We further impose the condition

$$
\tau_{q+3}^{(n)}=\tau_{q}^{(n)}, \quad q=1,2,3
$$

in (4.2). This simplifies the correspondence of these functions with the triangles of the octahedron in Fig. 2,

$$
\tilde{S}_{4}=(X, Y, Z)=\left(\tau_{1}^{(n-1)}, \tau_{3}^{(n-1)}, \tau_{2}^{(n-1)}\right), \quad S_{4}=\left(X^{\prime}, Y^{\prime}, Z^{\prime}\right)=\left(\tau_{3}^{(n)}, \tau_{1}^{(n)}, \tau_{2}^{(n)}\right) .
$$

Notice that (3.2) defines a map

$$
\left(\tau_{1}^{(n-1)}, \tau_{2}^{(n-1)}, \tau_{3}^{(n-1)}\right) \rightarrow\left(\tau_{1}^{(n)}, \tau_{2}^{(n)}, \tau_{3}^{(n)}\right)
$$

Therefore the behavior of the chain (3.4) will become explicit via iteration of the map started from arbitrary initial values $\left(\tau_{1}^{(1)}, \tau_{2}^{(1)}, \tau_{3}^{(1)}\right)$. 


\subsection{Localization}

In order to solve (4.2), it is convenient to study a localized version of the map by introducing new variables,

$$
\left(x_{1}^{(n+1)}, x_{2}^{(n+1)}, x_{3}^{(n+1)}\right):=\left(\frac{\tau_{1}^{(n-1)} \tau_{1}^{(n)}}{\tau_{3}^{(n-1)} \tau_{2}^{(n)}}, r \frac{\tau_{2}^{(n-1)} \tau_{2}^{(n)}}{\tau_{1}^{(n-1)} \tau_{3}^{(n)}}, \frac{\tau_{3}^{(n-1)} \tau_{3}^{(n)}}{\tau_{2}^{(n-1)} \tau_{1}^{(n)}}\right), \quad n \geq 2
$$

Then Eq. (4.2) is simplified significantly and we obtain the three-dimensional Lotka-Volterra equation $[7,8]$

$$
\begin{aligned}
& x_{1}^{(n+1)}=x_{1}^{(n)} \frac{1-x_{2}^{(n)}+x_{2}^{(n)} x_{3}^{(n)}}{1-x_{3}^{(n)}+x_{3}^{(n)} x_{1}^{(n)}}, \\
& x_{2}^{(n+1)}=x_{2}^{(n)} \frac{1-x_{3}^{(n)}+x_{3}^{(n)} x_{1}^{(n)}}{1-x_{1}^{(n)}+x_{1}^{(n)} x_{2}^{(n)}}, \\
& x_{3}^{(n+1)}=x_{3}^{(n)} \frac{1-x_{1}^{(n)}+x_{1}^{(n)} x_{2}^{(n)}}{1-x_{2}^{(n)}+x_{2}^{(n)} x_{3}^{(n)}} .
\end{aligned}
$$

This map has two invariants

$$
r=x_{1} x_{2} x_{3}, \quad s=\left(1-x_{1}\right)\left(1-x_{2}\right)\left(1-x_{3}\right),
$$

where $\left(x_{1}, x_{2}, x_{3}\right)$ denotes the initial point $p^{(0)}=\left(x_{1}^{(0)}, x_{2}^{(0)}, x_{3}^{(0)}\right)$. It was shown in [16-19], [20] that periodic points of all periods of this map form algebraic varieties. Since the varieties are determined by invariants alone we called them "IVPP". If $\gamma^{(n)}(r, s)=0$ denotes the IVPP of period $n$, they are given as follows:

$$
\begin{aligned}
\gamma^{(2)}(r, s)= & s+1 \\
\gamma^{(3)}(r, s)= & (r-s)^{2}+(r+1)(s+1) \\
\gamma^{(4)}(r, s)= & -(r-s)^{3}+s(r+1)^{3} \\
\gamma^{(5)}(r, s)= & (r-s)^{6}-(r-2)(r+1)(s+1)(r-s)^{4} \\
& -(2 s+r)(r+1)^{2}(s+1)^{2}(r-s)^{2}+s^{2}(r+1)^{3}(s+1)^{3}, \\
\gamma^{(6)}(r, s)= & -3(r-s)^{4}-(r+1)(s+1)((r+1)(s+1)-3)(r-s)^{2} \\
& -3(r+1)(s+1)\left(r^{3}+s^{3}-r^{2} s^{2}-2 r^{2} s-2 r s^{2}-r^{2}-s^{2}\right),
\end{aligned}
$$

\subsection{Singularity confinement}

The singularity confinement is a phenomenon of a map, which is mapped once to infinity, and then returns to a finite point after some steps $[5,12,13]$. We have shown in [19] that, if we continue the map (4.5) after it is recovered from the singularity, IVPPs (4.6) are generated iteratively. 
More precisely, suppose we start the map (4.5) from a point $p^{(0)}$ in

$$
\Sigma_{+}:=\left\{x_{1}, x_{2}, x_{3} \mid 1-x_{3}+x_{3} x_{1}=0 \cup 1-x_{1}+x_{1} x_{2}=0 \cup 1-x_{2}+x_{2} x_{3}=0\right\},
$$

such that one of the denominators of $p^{(1)}=\left(x_{1}^{(1)}, x_{2}^{(1)}, x_{3}^{(1)}\right)$ vanishes. The initial point $p^{(0)} \in \Sigma_{+}$can be expressed by the invariants as

$$
p^{(0)} \in\left\{\left(\frac{r-s}{r+1}, r \frac{s+1}{r-s}, \frac{r+1}{s+1}\right) \cup\left(\frac{r+1}{s+1}, \frac{r-s}{r+1}, r \frac{s+1}{r-s}\right) \cup\left(r \frac{s+1}{r-s}, \frac{r+1}{s+1}, \frac{r-s}{r+1}\right)\right\}
$$

and is mapped by (4.5) to

$$
p^{(1)} \in\{(\infty, 0,1) \cup(1, \infty, 0) \cup(0,1, \infty)\}
$$

and then to

$$
p^{(2)} \in\{(1,0, \infty) \cup(\infty, 1,0) \cup(0, \infty, 1)\} .
$$

In the next step it returns to a finite point

$$
p^{(3)} \in\left\{\left(\frac{r+1}{s+1}, r \frac{s+1}{r-s}, \frac{r-s}{r+1}\right) \cup\left(\frac{r-s}{r+1}, \frac{r+1}{s+1}, r \frac{s+1}{r-s}\right) \cup\left(r \frac{s+1}{r-s}, \frac{r-s}{r+1}, \frac{r+1}{s+1}\right)\right\} .
$$

Notice that $\gamma^{(2)}=s+1$ appears in the denominator of one of $x_{j}^{(3)}$ 's. This is expected because a period 2 point must diverge at the same point after two steps of the map if it is recovered from the singularity. If we continue the map further we always find $\gamma^{(n)}$ in the denominator of $x_{j}^{(n+1)}$ if $x_{j}^{(1)}$ was divergent.

We can rephrase this result in terms of $\tau$ 's. Namely, assuming $\left(\tau_{1}^{(1)}, \tau_{2}^{(1)}, \tau_{3}^{(1)}\right)=(1,1,1)$, and comparing (4.4) and (4.7), we see

$$
\left(\tau_{1}^{(2)}, \tau_{2}^{(2)}, \tau_{3}^{(2)}\right) \in\{(r+1, s+1, r-s) \cup(r-s, r+1, s+1) \cup(s+1, r-s, r+1)\}
$$

In this form one of $\left(\tau_{1}^{(2)}, \tau_{2}^{(2)}, \tau_{3}^{(2)}\right)$ is $s+1$, i.e. vanishes on the IVPP of period 2 . As we repeat the map it is easy to convince ourselves that

$$
\tau_{j}^{(2)}=\gamma^{(2)} \Rightarrow \tau_{j}^{(n)} \propto \gamma^{(n)}(r, s), \quad n \geq 3
$$

a phenomenon compatible with the conjecture of [13] for the HM equation. In this way the iteration of the localized map (4.5) generates IVPPs of all periods if the initial point is chosen on $\Sigma_{+}$.

Finally we summarize the arguments in this section by the words of the theory of category. The notion of localization of category is important to consider a derived category. By a proper reduction of the complex (3.4), we defined the localization of the complex explicitly by (4.4). This procedure will not be interesting at all if a zero of the denominator remains zero as we apply the shift (or the map). Remarkably this is not the case, if the objects are the $\tau$ functions. In this particular case the singularity confinement takes place. After the recovery from the divergence we find nonzero $\tau$ functions, such as (4.9), which are polynomial of the local coordinates defined by (4.4). Moreover zeros of these polynomials determine periodic points of the map, i.e. the IVPPs. Therefore we conclude naturally that the set of IVPPs of this map is nothing but the generating system of the category. 


\section{Conclusion}

We have shown how the theory of KP hierarchy is embedded within the theory of derived category. From our investigation it is clear that the integrability of the maps is guaranteed by the octahedral axioms. In particular the generation of algebraic varieties (IVPP's) from the singularity confinement should provide various information about the nature of the category.

The present note is certainly not complete from mathematical point of view. We would like to supply the gap in our forthcoming paper.

\section{Acknowledgments}

This work was done two years ago in collaboration with Noriyasu Kozako at Tokyo Metropolitan University. The author would like to thank him who introduced the theory of derived category to the author. A large part of mathematical idea owes to him, although details of their description have not been given in this note.

\section{References}

[1] V. E. Adler, A. I. Bobenco and Yu. B. Suris, Classification of integrable discrete equations of octahedron type, Int. Math. Res. Not. 2012 (2012) 1822-1889.

[2] P. S. Aspinwall, D-Branes on Calabi-Yau Manifolds, in Progress in String Theory: TASI 2003 Lecture Notes (World Scientific, 2005), arXiv:hep-th/0403166.

[3] A. Caldararu, Derived categories: A skimming, in Snowbird Lectures in Algebraic Geometry, Contemporary Mathametics, Vol. 388 (American Mathematical Society, Providence, RI, 2005), pp. $43-75$.

[4] S. I. Gelfand and Yu. I. Manin, Methods of Homological Algebra, 2nd edn., Springer Monographs in Mathematics (Springer-Verlag, Berlin, 2003).

[5] B. Grammaticos, A. Ramani and V. Papageogiou, Do integrable mappings have the Painlevé property? Phys. Rev. Lett. 67 (1991) 1825-1828.

[6] R. Hirota, Discrete analogue of a generalized Toda equation, J. Phys. Soc. Jpn. 50 (1981) $3787-3791$.

[7] R. Hirota and S. Tsujimoto, Conserved quantities of a class of nonlinear difference-difference equations, Phys. Soc. Jpn. 64 (1995) 3125-3127.

[8] R. Hirota, S. Tsujimoto and T. Imai, Difference scheme of soliton equations, in Future Directions of Nonlinear Dynamics in Physical and Biological Systems, eds. P. L. Christiansen et al. (Plenum Press, New York, 1993), p. 7.

[9] M. Kontsevich, Homological algebra of mirror symmetry, in Proc. Int. Cong. Mathematicians (Birkhäuser, 1995), pp. 120-139, arXiv:alg-geom/9411018.

[10] N. Kozako, On the derived category of quasi-coherent sheaves over an algebraic variety and its compact generator, a thesis presented to the Division of Natural Sciences, Graduate School of International Christian University (2009).

[11] T. Miwa, On Hirota's difference equations, Proc. Japan Acad. A 58 (1982) 9-12.

[12] A. Ramani, B. Grammaticos and J. Hietarinta, Discrete version of Painlevé equations, Phys. Rev. Lett. 67 (1991) 1829-1832.

[13] A. Ramani, B. Grammaticos and J. Satsuma, Integrability of multidimensional discrete systems, Phys. Lett. A 169 (1992) 323-328.

[14] S. Saito, String theories and Hirota's bilinear difference equation, Phys. Rev. Lett. 59 (1987) $1798-1801$.

[15] S. Saito and N. Saitoh, Gauge and dual symmetries and linearization of Hirota's bilinear equations, J. Math. Phys. 28 (1987) 1052-1055.

[16] S. Saito and N. Saitoh, Invariant varieties of periodic points for the discrete Euler top, SIGMA 2 (2006), paper 098, 10 p., http://www.emis.de/journals/SIGMA. 
[17] S. Saito and N. Saitoh, Invariant varieties of periodic points for some higher dimensional integrable maps, J. Phys. Soc. Jpn. 76 (2007) 024006, http://jpsj.ipap.jp/link?JPSJ/76/024006.

[18] S. Saito and N. Saitoh, On recurrence equations associated with invariant varieties of periodic points, J. Phys. A: Math. Theor. 40 (2007) 12775-12787.

[19] S. Saito and N. Saitoh, Fate of the Julia set of higher dimensional maps in the integrable limit, J. Math. Phys. 51 (2010) 063501.

[20] N. Saitoh and S. Saito, Perturbative changes of the nature of invariant varieties for some higher dimensional integrable maps, J. Phys. Soc. Jpn. 77 (2008) 024001.

[21] N. Shinzawa and S. Saito, A symmetric generalization of linear Bäcklund transformation associated with the Hirota bilinear difference equation, J. Phys. A: Math. Gen. 31 (1998) 4533-4540. 